\title{
5. Ueber
}

\section{die Veruendung hochfrequenter Wechselströme zum Studium electrischer Gasentladungen ${ }^{1}$ ); von $\boldsymbol{H}$. Ebert.}

(Mittheilang aus dem physik. Institute der k. Technischen Hochschule $\mathrm{zu}$ München.)

Die electrischen Leitungswiderstände der Gase und dauit die Transformation electrischer Energie in andere Energieformen, namentlich in Wärme, bei dieser Klasse von Leitern haben von jeher dem Verständnisse grosse Schwierigkeiten dargeboten. Man hat sich schliesslich zu der Ueberzeugung hingedrängt gefühlt, dass wir hier überhaupt kein einfaches Phänomen vor uns haben, sondern dass der Energieumsatz in einem Entladungsrohre in mehreren zwar gleichzeitig nebeneinander herlaufenden, aber ihrer inneren Natur nach doch sehr verschiedenen Processen besteht, eine Auffassung, welche durch Beobachtungen über die Verschiedenheit des Potentialabfalles und der Wärmeentwickelung in den einzelnen Theilen des Rohres wesentliche Stützen findet. Es war daher nicht möglich, ein vom electrischen Strome durchflossenes Gas mit einem Leiter I. Klasse oder einem solchen II. Klasse unmittelbar zu vergleichen; in der That sind alle Versuche fehlgeschlagen, hier ähnlich einfache Leitungsgesetze, wie sie für diese gelten, aufzufinden. Fasst man die Andeutungen, welche hier und da erhalten worden sind, zusammen, so kommt man zu dem Schlusse, dass sich eine leitende Gassäule wie die Uebereinanderlagerung zweier Gebilde verhält:

1) Die vorliegende Untersuchung wurde mit den Hülfsmitteln des Elizabeth Thompson science fund zu Boston angestellt; es sei mir gestattet dem Board of Trusties dieses Fund auch an dieser Stelle meinen ergebensten Dank auszusprechen. Die von mir benutzte Wechselstrommaschine wurde von Hrn. Ingenieur Hummel in München in dankenswerthester Weise zur Verfügung gestellt. 
1. eines Leiters, der nach Art der gewöhnlichen Leiter I. Klasse oder aber auch der Electrolyte den Electricitätsübergang vermittelt, für den vielleicht ein dem $0 \mathrm{hm}$ 'schen Gesetze entsprechendes einfaches Gesetz gilt, und bei dem ein Theil der Stromenergie nach einem dem Joule'schen ähnlichen Gesetze in Wärme verwandelt wird;

2. eines Capacitätswiderstandes, herrührend von einer Art dielectrischen Zwangszustandes, der wie ein Polarisationswiderstand wirkt, welchen die Spannung erst überwunden haben muss, ehe die Entladung überhaupt eintritt, und der mit einer nicht zu vernachlässigenden Polarisationscapacität ausgerüstet ist. Man geht gewiss nicht irre, wenn man den Sitz dieses dielectrischen $\mathrm{Z}$ wangszustandes wesentlich in der Nähe der Elektroden sucht. Da Strom durch die polarisirte Zwischerschicht selbst hindurchgeht, so gleicht das Entladungsrohr einem „durchschlagenen Condensator“. Ausser Joule'scher Wärme wird noch u. A. infolge der dielectrischen Hysteresisarbeit Wärme entwickelt, das Phänomen erhält dadurch eine grössere Zahl ron Bestimmungsstücken.

In ähnlicher Weise ist der Entladungsvorgang sowohl in früheren wie in neueren Arbeiten gelegentlich aufgefasst worden; ich wollte versuchen, hierüber weitere Anhaltspunkte zu gewinnen. Die Wechselstromtheorie hat das Problem der Messung. an einem Condensator mit Stromdurchgang (mit "leakage", "Lecksein") schon vor längerer Zeit gelöst. Bei Anwendung von Wechselstrom musste jene Capacitätswirkung besonders deutlich zu Tage treten, da die zur Ladung nöthigen Energiebeträge bei jeder Entladung wieder zurückerstattet werden, während die durch den hindurchgehenden Strom hervorgerufene Wärme zusammen mit allen anderen auf sogenannte „electrische Reibung" verwendeten Beträgen im Wattmeter zum Ausdruck kommt. Wurde daher die Wechselzahl nur genügend hoch gewählt, so musste der erste Betrag merkbare Werthe neben dem zweiten erhalten. Dies war ein erster Gesichtspunkt, von dem aus ich den Versuch machte, das Verbalten der Gasentladungen in hochfrequenten Wechselstromfeldern quantitativ zu untersuchen. Die mitgetheilten Messungen beziehen sich zunächst nur auf einzelne Fälle und sollen vorerst lediglich die Anwendbarkeit der Methode erläutern, sowie 
ein Urtheil über die Grössenordnung der zu messenden Werthe liefern; bei späterer Gelegenheit gedenke ich ein ausführlicheres Beobachtungsmaterial und seine Discussion zu veröffentlichen. Die Verwendung verhältnissmässig schwacher, aber hochfrequenter Wechselströme zum Studium der Gasentladungen schien mir aber noch aus anderen Gründen empfehlenswerth:

Durch eine Reihe neuerer Experimentaluntersuchungen ist gezeigt worden, dass die electrischen Entladungen durch verdünnte Gase hindurch besonders bemerkenswerthe Formen annehmen, wenn die Erregung durch rasch verlaufende electrische Schwingungen von genügender Amplitude erfolgt. In grossem Maassstabe wurden solche Versuche zuerst von Nikolas Tesla angestellt mit eigens hierzu construirten vielpoligen Maschinen mit einer sehr grossen Wechselzahl. ${ }^{1}$ ) Später verliess Tesla diese directe Erzeugung des hochfrequenten Wechselstromes durch einen Generator und verwendete die oscillatorisch verlaufenden Entladungsströme grosser Condensatoren, welche eine Transformation auf sehr hohe Potentiale gestatteten; die Ladungen erfolgten durch gewöhnlichen Wechselstrom. Himstedt zeigte, dass man die wesentlichsten der von Tesla beobachteten Erscheinungen erhält, wenn man die Condensatoren mittels eines genügend grossen Inductoriums ladet; die meisten Untersuchungen über die Teslaerscheinungen arbeiten mit der Himstedt'schen Anordnung. ${ }^{2}$ ) Condensatorschwingungen Leydener Flaschen wurden ferner bei den Versuchen von J.J. Thomson und d'Arsonval verwendet. ${ }^{3}$ ) E. Wiedemann und H. Ebert benutzten die Schwingungen der Lecher'schen Drahtanordnung, indem sie die zu erregenden Gasräume in den Bereich des Endcondensators derselben brachten. ${ }^{4}$ )

1) Vgl. u. a. „Experimente mit Strömen hoher Wechselzahl und Frequenz" von Etienne de Fodor, revidirt und mit Anmerkungen versehen von $\mathrm{Nik}$ olas Tesla. Hartlebens Verlag 1894, sowie "Nikolas Tesla's Untersuchungen über Mehrphasenströme und über Wechselströme hoher Spannung und Frequenz" von Th. Commerford Martin, deutsch von H. Maser. Verlag von W. Knapp 1895.

2) F. Himsiedt, Ber. d. Oberhess. Gesellsch. Giessen. 30. Sep.Abdr. 14 pp. 1894.

3) J. J. 'Thomson, Phil. Mag. (5) 32. p. 321 u. 445. 1891; Wied. Beibl. 16. p. 560. 1892.

4) H. Ebert u. E. Wiedemann, Wied. Ann. 48. p. 591; 49. p. 1 u. $32 ; 50$. p. 1 u. 221.1893. 
Charakteristisch für das Aussehen der Gasentladungen bei den genannten Erregungsformen ist vor allem das Ueberwiegen der Kathodenerscheinungen; dass auch das Leuchten der unipolaren Teslalampen auf Kathodeneffecten beruht, ist von P. Spiess nachgewiesen worden. ${ }^{1}$ ) Wiewohl die Richtung des die Gassäule durchbrechenden Stromes fortwährend wechselt, gelangen bei allen diesen Anordnungen an den Electroden oder tuberbaupt an den den Gasraum begrenzenden Wänden scheinbar nur die Dunkelräume und Glimmlichtschichten beiderseitig zur Ausbildung und die Anodenerscheinung tritt beinahe ganz zurück. Ferner treten Kathoden-, Lenard- und Röntgenstrahlen schon bei viel höheren Drucken und in erheblich stärkerem Grade unter den Wirkungen rascher electrischer Schwingungen (namentlich bei starkem Curvenabfalle) auf, als bei einseitig gerichteten Einzelentladungen. EigenthümlicheImpedanzphänomene electroluminescirender Gassäulen, welche $\mathrm{T} \theta \mathrm{sla}$ beschrieb, bedürfen wohl noch der näheren Aufklärung.

Die Erregung durch Condensatorschwingungen hat aber den Nachtheil, dass diese Schwingungen sehr rasch abklingen, die einzelnen aufeinanderfolgenden Stromphasen also äusserst ungleichwerthig sind. Durch Abstimmen aufeinander resonirender Leiterkreise (E. Wiedemann und H. Ebert) oder durch gleichzeitige Verminderung der Capacitäten und Erhöhung der Selbstinductionen (H. Ebert) kann man zwar den jeder Hauptentladung folgenden Schwingungszug, der auf das verdünnte Gas wirkt, nicht unerheblich verlängern; doch schliessen sich auch hier den Epochen der Erregung verhältnissmässig lange Zeiträume an, in denen das Gas nicht erregt wird.

Die Erfahrung, dass ein einmal zum Leuchten und damit zum Leiten angeregtes Gas der unmittelbaren Wiedererregung viel leichter zugänglich ist, als wenn erst eine längere Periode der Nichterregung über dasselbe hingegangen ist, legte den Wunsch nahe, zum näheren, ñamentlich auch quantitativen Studium der obengenannten Phänomene eine Érregung durch eine ununterbrochene Folge einander vollkommen äqui-

1) P. Spiess, Inaug.-Diss. p. 46. Kiel 1896. 
valenter Schwingungsphasen, also durch einen hochfrequenten Wechselstrom zur Verfügung zu haben. Schon frühere Versuche hatten erkennen lassen, dass bei Anwendung harmonischer Hochfrequenzströme die Abnutzung des Electrodenmateriales eine weit geringere, als bei der Verwendung der Condensatorentladungen ist. ${ }^{1}$ ) Ferner liefert eine gute Wechselstrommaschine bei gleichmässiger Felderregung ausserordentlich constante Bedingungen; eine grosse Entladungszahl ist leicht herzustellen, und man umgeht alle die Schwierigkeiten, welche bei den Inductorien die Unterbrecher immer in die Anordnung hineinbringen.

Ein Zurückgreifen auf' die ursprüngliche Tesla'sche Anordnung war nicht angezeigt. Die Teslamaschinen arbeiten mit einer ausserordentlich grossen Wattzahl. Misst man aber die Energie, welche von einem selbst in heftigster Luminescenzstrahlung begriffenen verdünnten Gase consumirt wird, so erkennt man, dass dieselbe äusserst gering ist. Es musste daher auch umgekehrt möglich sein, das Luminesciren eines solchen Gases durch Zuführung einer sehr geringeu Wattzahl zu unterhalten, wenn man nur die Erregungsbedingungen geeignet wählte. Man gewinnt dabei gleichzeitig noch verschiedene andere Vortheile. Die Erwärmung der Electroden und der Röhrenwände und damit mittelbar des Gases selbst, bleibt eine geringere; in dem Electrodenuateriale occludirte, an den Wänden adsorbirte störende Gasreste werden nicht so leicht losgerissen und in Freiheit gesetzt, der Gasinhalt bleibt also während einer längeren Zeit identisch. Letzteres ist aber unbedingt nothwendig, wenn man genau vergleichbare Beobachtungen anstellen will.

Dies waren die Gesichtspunkte, welche Hrn. Hummel und mich selbst nach einigen orientirenden Vorversuchen in dem Versuchslaboratorium der vormals Schuckert'schen Electricitätswerke zu Nürnberg im Jahre 1894 veranlassten, ein neues System zur Untersuchung von Gasentladungen unter der Wirkung electrischer Schwingungen auszuarbeiten. Maassgebend war vor allem auch das Ziel, die Anordnung so zu treffen,

1) Vgl. u. a Etienne de Fodor, Experimente mit Strömen hoher Wechselzahl und Frequenz. p. 218. 1894. 
dass alle einzelnen, die Erscheinungen bedingenden Factoren einer exacten Messung zugänglich gemacht werden konnten.

Ich erlaube mir im Folgenden die Maschine sowie alle dazu gehörigen Hülfsanordnungen $\mathrm{zu}$ beschreiben und die Wirkungsweise des neuen Hülfsmittels an Beispielen zu erläutern; zugleich möchte ich Hrn. Ingenieur G. Hummel in München meinen besten Dank sagen, der keine Mühe gescheut hat, nach vielfachen Versuchen und Abänderungen und mit steter Rücksichtnahme auf die Wünsche und Bedürfnisse des Experimentirenden den sogleich zu beschreibenden.Generator zu bauen, der auch als höchst compendiöser Motor verwendbar ist.

\section{Der Generator.}

Zunächst war festzustellen, wie hoch die Wechselzahl zu steigern sei, damit die charakteristischen zweiseitigen Kathodenerscheinungen und die bemerkenswerthe Symmetrie der ganzen Gasentladung, welche für die Erregung durch reine Schwingungen bezeichnend ist, auftreten. Bei einer Reihe von Vorversuchen mit einem kleineren Modelle ${ }^{1}$ ) der weiter unten zu beschreibenden Wechselstrommaschine stellte sich heraus, dass man mit der Frequenz gar nicht so hoch zu steigen brauche, als anfänglich erwartet wurde, sondern dass ca. 1000 Zeichenwechsel pro Secunde ausreichend sind, während die Schwingungszahlen bei Condensatorentladungen nach Hunderttausenden, bei der Lecher'schen oder Blondlot'schen Anordnung nach Millionen rechnen.

Bei dieser Wechselzahl von 1000 pro Secunde wurden alle von E. Wiedemann und $\mathrm{H}$. Ebert ${ }^{2}$ ) beschriebenen Erscheinungen der verdünnten Gase in einem hochfrequenten electrischen Schwingungsfelde erhalten, der viel grösseren Energie entsprechend, sogar noch viel glänzender; auch die bemerkenswerthen Erscheinungen, welche in dem Gasraum isolirt aufgestellte metallische Leiter zeigen ${ }^{2}$ ), bei denen die Uebereinanderlagerung zeitlich nacheinander ausgebildeter Glimmlichter zum Entstehen einer positiven Lichtbrücke Ver-

1) Dasselbe ist ebentalls von Hrn. Hummel gebaut und Hrn. E. Wiedemann überlassen worden, dern ich für die freundliche Leihung desselben auch an dieser Stelle meinen besten Dank ausspreche.

2) H. Ebert a. E. Wiedemann, Wied. Ann. 62. p. 174. 1897. 
anlassung zu geben scheinen, werden hier mit grossem Detailreichthum erhalten.

Die Vortheile, die man durch Beschränkung der Wechselzahl erreicht, liegen ausser auf constructivem Gebiete noch besonders darin, dass man den zeitlichen Verlauf der ganzen Erscheinung im Drehspiegel bequem verfolgen kann, was bei den hochfrequenten Condensatorentladungen kaum möglich ist. Setzt man den Drehspiegel direct auf die Axe der Maschine, so kann man hier je nach der Richtung, in der man in denselben blickt, einen Einblick in jede einzelne Phase der Entwickelung der Lichterscheinung erhalten; denn diese sind immer an bestimmte Stellungen des Maschinenankers gebunden. Will man die Wirkung mebrerer aufeinander folgender Schwingungen miteinander vergleichen, so stellt man einen Drehspiegel mit besonderem Antriebe neben die Entladungsröhre. Schon bei leicht zu erreichenden Tourenzahlen erhält man eine völlig klare Auflösung der ganzen Erscheinungsreihe.

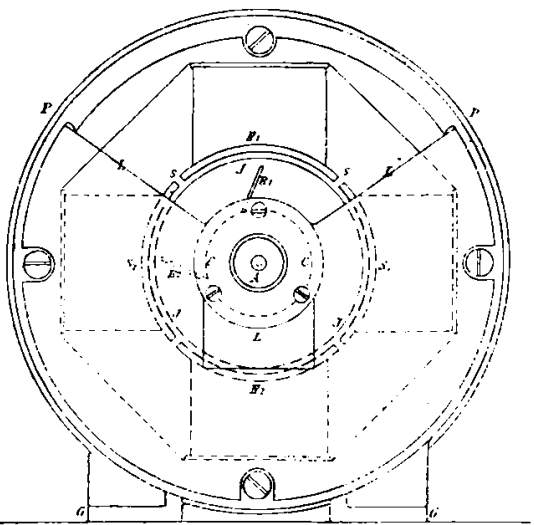

Fig. 1.

Der Erzeuger des hochfrequenten Wechselstromes gehört zu dem Typus der sogenannten Gleichstromwechselstrom-Transformer. Er wird mit Gleichstrom von etwa 55-64 Volt Spannung an den Klemmen der Maschine und 1,2-1,9 Ampère Stromstärke beschickt, bei der gewöhnlich verwendeten Belastung (vgl. weiter unten). Fig. 1 stellt die kleine Maschine schematisch in Vorderansicht (in $1 / 3$ natürlicher Grösse) dar. Das aus aufeinander geschichteten Eisenlamellen bestehende Polgehäuse P.P trägt unten die Grundplatte $G G$, nach innen vier radial verlaufende geblätterte Feldmagnete von kurzer gedrungener Gestalt, welche die Magnetisirungsspulen tragen. Dieselben sind paarweise neben- und die beiden Gruppen hintereinander so geschaltet, dass ein vierpoliges magnetisches Feld entsteht. Jeder Magnet trägt 10 Lagen von je 18 Windungen der 0,8 mm 
dicken Erregerwickelung; der gesammte Widérstand der so angeordneten Feldwickelung beträgt 1,81 $\Omega$. Innerhalb der bei Speisung mit dem Gleichstrome sich ausbildenden gesonderten vier magnetischen Kreise von erbeblichem Inductionsflusse rotirt der Inductor $J J J$, ein Trommelanker mit Serienwickelung.

Ueber die Enden der vier Feldmagnete sind geeignet ausgeschnittene übergreifende Bleche $s s$ aus Eisen geschoben, welche zwischen sich nur schmale Unterbrechungen übrig lassen. Dabei steht die Breite der Pole und der Spulenwickelung zu dem Abstand der Mitten zweier benachbarter Pole in einem solchen Verhältniss, dass ein möglichst reiner sinoidaler Stromverlauf erzielt wird.

Die Feldmagnete können sowohl in Serie mit der Ankergleichstromwickelung gelegt, als auch gesondert gespeist werden; gewöhnlich geschah das erstere.

Die durch Glimmerzwischenlagen voneinander gut isolirten Ankerbleche enthalten am Rande 38 Einschnitte, in denen die 19 Abtheilungen der rechteckig gestalteten Windungen, acht innerhalb einer jeden Nuht, liegen. Die Enden der Abtheilungen sind nach den Lamellen des Collectors $C C$ geführt. Von dieser Wickelung für den Gleichstrom, der die Maschine in Umdrehung versetzt, wird der Wechselstrom nach zwei Schleifringen an der Rückseite der Maschine abgezweigt.

Die Axe $A$ wird von einem Lagerbock $L L L$ getragen, welcher als halbkugelförmige Schale direct an das Polgehäuse $P P$ angeschraubt und nur oben geöffnet ist. Dem in der Figur gezeichneten Lagerbock entspricht ein gleicher auf der Rückseite. Dadurch wird nicht nur ein sehr hoher Grad von Stabilität erreicht, sondern die inneren Theile der Maschine liegen auch sehr geschützt. Der vordere Lagerbock trägt innen einen Ansatz, auf dem der die Bürstenhalter $B_{1} B_{2}$ tragende Ring sich bewegen lässt.

Die Lagerschalen sind kugelförmig ausgedreht, in ihr ruhen die beiden Kugellager, welche eine freie Drehbarkeit der Lagerbuchse ermöglichen.

Für eine möglichst vollkommene Ausbalancirung und eine sichere Bandagirung der Trommelwindungen, sowie eine gute automatische Schmierung der Lager (Ringschmierung) wurde 
bei den erstrebten hohen Touren gan\% besonders Sorge getragen. Bei Leerlauf ergaben sich folgende Tourenzahlen:

Bei Betrieb mit

Gleichstrom ron

2s Volt 5000 Touren in der Minute.

43 " $10000, ", "$,

$60 " 16000, ", ", "$,

$75,20000, ", "$,

Bei der gewöhnlich vorhandenen Belastung hatte die Trommel $J J J$ von $5 \mathrm{~cm}$ Durchmesser eine mittlere Umdrehungszahl von 180 bis 200 in der Secunde, mithin eine Umfangsgeschwindigkeit bis zu $31 \mathrm{~m} / \mathrm{sec}$. Da die sonst angewandte Peripheriegeschwindigkeit bei Dynamomaschinenankern die Zahl von $15-20 \mathrm{~m} / \mathrm{sec}$ nur selten übersteigt, so erkennt man, dass hier in der That besondere Maassnahmen getroffen werden mussten.

Auf dem vorderen Theil der Axe, der etwas aus dem Lager hervorragte, wurde ein kleiner Stahldrehspiegel von ca. $1 \frac{1}{\mathrm{~cm}^{2}}$ polirter Fläche direct aufgesteckt; seine Masse war völlig symmetrisch um die Axe vertheilt, sodass durch ihn kein Schleudern herbeigeführt wurde. Hr. Hummel hat zu der Maschine noch ein sehr leichtes, aber höchst stabiles Vorgelege gebaut, dessen Scheiben ein Durchmesserverhältniss von 2:10 haben. Die Uebersetzung der Drehgeschwindigkeiten ist naturgemäss nicht ganz eine fünffache, da bei diesen grossen Tourenzahlen erhebliche Gleitungsverluste eintreten. Immerhin eignet sich die Maschine also auch zur Messung ausserordentlich kleiner Zeitintervalle, z. B. bei der Bestimmung der Fortpflanzungsgeschwindigkeit des Lichtes, der electrischen Entladung oder der Kathoden-, Lenard- oder Röntgenstrahlen. Die Umdrehungszahl der Maschine kann ziemlich sicher aus der Höhe des pfeifenden Tones, den sie giebt, durch Vergleich mit einer Zählsirene bestimmt werden.

Der beschriebene Transformer ruht auf Kautschuk und ist völlig in einen Kasten eingeschlossen, der nur ein seitliches Fenster zur Controle der Bürsten hat. Die Zuleitungen des Feld- und Ankergleichstromes sowie die Ableitungen des Wechselstromes sind isolirt durch den Boden des abhebbaren Kastens geführt. 
Die Wechselstromniederspannung, welche die Maschine direct gab, betrug 32-35 Volt (effectiv), bei Stromstärken von 0,6 bis 1,5 effectiven Ampère (je nach der Belastung). Um die Gassäulen zu durchbrechen, musste dieser Strom etwa auf die 30-40 fachen Spannungen transformirt werden. An Stromstärke bleibt dann noch immer genügend übrig.

\section{Der Transformator.}

Eine gewisse Schwierigkeit bietet die Transformation der hochfrequenten Ströme auf hohe Spannungen. Der Transformator muss bis $\mathrm{zu}$ einem gewissen Grade den speciellen Zwecken, zu dem man den Hochfrequenzstrom verwenden will, angepasst sein. Vor allem darf die Spannungscurve des transformirten Stromes nicht zu flach ausfallen, weil ein verdünntes Gas, welches zum Leuchten angeregt werden soll, einen bestimmten, hauptsächlich ron seinem Drucke abhängigen Spannungsgradienten erfordert.

Tesla verwendete bei seinen Maschinen völlig eisenfreie Transformatoren. Eine grössere Sammlung von Kraftlinien war aber im vorliegenden Falle nothwendig. Nach orientirenden Vorversuchen mit einem kleinen Ringtransformator mit einem Mantel von Eisendraht, den mir Hr. Hummel freundlichst zur Verfügung stellte, wurden hauptsächlich Igeltransformatoren von gestreckter, kurzer Form mit offenem Eisenkreise verwendet; durch gute Untertheilung des Eisenkernes wurden die Störungen durch Wirbelströme möglichst herabgesetzt. Der eine Transformator $a$ hatte folgende Dimensionen:

Ueber ein $28 \mathrm{~cm}$ langes, $3 \mathrm{~cm}$ dickes Bündel von $1 \mathrm{~mm}$ starken, gut ausgeglühten lackirten Eisendrähten ist die Primärspirale von je 278 Windungen in 4 Lagen, also zusammen 1112 Windungen eines $0,75 \mathrm{~mm}$ dicken Kupferdrahtes gewickelt; der Widerstand dieser Spule wurde zu $2,23 \Omega$, die Selbstinduction nach der Maxwell-Dorn'schen Methode zu 0,040 Erdquadranten bei der angewendeten Stromstärke bestimmt.

Die Windungszahl der secundären Spirale liess sich leider nach der Anfertigung nicht mehr direct bestimmen; ihr Widerstand wurde zu angenähert $9990 \Omega$ gefunden. Ihr Selbstinductionscoefficient ergab sich (mit Eisenkern) zu rund 212 Erdquadranten. Das Transformationsverhältniss war etwa 1:50. 
Ein anderer T'ransformator $b$ von ganz ähnlicher Form, der bei einigen Versuchsreihen an Stelle von $a$ verwendet wurde und sich als noch günstiger erwies, hatte einen Eisenkern, welcher aus $0,8 \mathrm{~mm}$ dickeu, ausgeglühten Drähten von $53 \mathrm{~cm}$ Länge hergestellt war und eine Dicke von $3,2 \mathrm{~cm}$ hatte. Die Windungslänge der primären Wickelung betrug $27 \mathrm{~cm}$; die Wickelung bestand aus 2 Lagen von je 220 Windungen. Der Widerstand der Niederspannungswickelung betrug 1,142 $\Omega$, derjenige der Hochspannungswickelung $13550 \Omega$.

Wenn die Eisenverhältnisse bez. die Windungszahlen wesentlich andere waren, so wurde der grösste Theil der Primärenergie hauptsächlich für die Leistung der Hysteresisarbeit verwendet oder in Joule'sche Wärme verwandelt, für die Secundärleistung blieb dann nichts mehr übrig. Die weiter unten mitgetheilten Tabellen zeigen, wie gut im vorliegenden Falle die richtigen Verhältnisse getroffen waren, sodass nach der Transformation mehr als 80 Proc. der Hochfrequenzwechselstromleistung im secundären Kreise wieder gewonnen wurden, trotzdem der Transformator mit offenem magnetischen Kreise arbeitete und daher noch eine erhebliche Streuung besass.

Gelegentlich wurde auch mit einem Messtransformator der Doppel-E-Type mit völlig geschlossenem Eisenkreise gearbeitet von 100 primären und 2000 secundären Windungen, dessen Transformationsverhältniss also 1:20 war.

\section{Das Ampèremeter.}

Da das Transformationsverhältniss der Igeltransformatoren nur angenähert bekannt war, die Production der Maschine im Wechselstromkreise sich der Belastung anpasste, mit dieser also variirte, und die am leichtesten ausführbare Gleichstrommessung daher nur ein ungefähres Bild von der der Entladungsröhre wirklich zugeführten Secundärleistung geben konnte, so musste zum genaueren Studium der Anregungsbedingungen ein Messinstrument in Serie mit dem Entladungsrohre in den Secundärkreis des Transformators selbst aufgenommen werden. Einen so hochfrequenten und verhältnissmässig hochgespannten (bis ca. 2900 Volt) Wechselstrom direct zu messen, war nicht leicht, zumal die gesammte Energie so knapp bemessen war, dass nicht viel derselben durch die 
Messung selbst verloren gehen durfte. Vor allem musste jede irgendwie beträchtliche Inductanz vermieden werden, welche den ganzen Strom gedrosselt hätte, und auch wegen der Isolation musste jedes Bedenken gehoben sein. Von den zahlreichen Formen, die in Vorschlag gebracht worden sind, eignete sich nur eine, welche von Heinrich Hertz beschrieben worden ist, im Princip ein Hitzdrahtampèremeter. ${ }^{1}$ ) Vorversuche mit kleineren Apparaten, welche sich 'eng an die Hertz'sche Construction, namentlich in den Dimensionen anschlossen, zeigten, dass für den vorliegenden Zweck doch einige Varia-

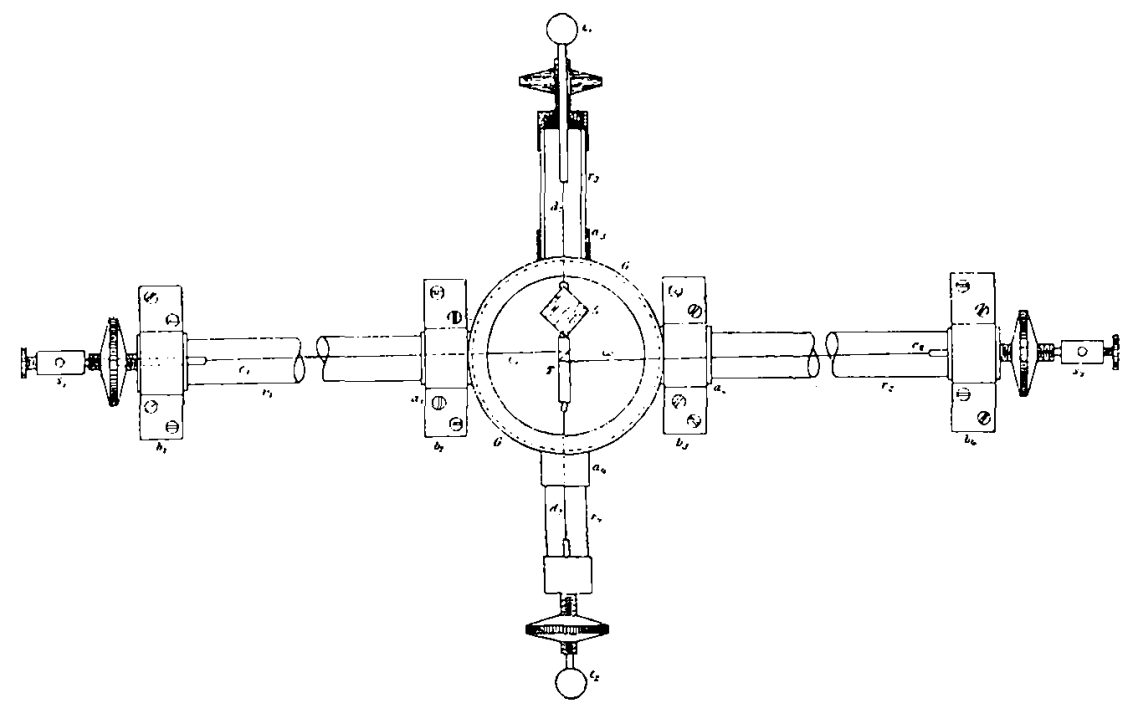

Fig. 2.

tionen nöthig waren; bei der Kleinheit der zu messenden Ströme (20-25 Milliamp̀ère im Maximum) mussten die Hitzdrähte länger sein. Dies erforderte wieder einen besonderen Schut/ gegen Luftströmungen und so entstand schliesslich das in Fig. 2 schematisch dargestellte Instrument.

An ein rückwärts mit einem Hartgummideckel, nach vorn durch eine Spiegelglas- oder Glimmerplatte geschlossenes Gebäuse $G G$ aus Ebonit sind vier kurze Rohrstutzen aus Hart-

1) H. Hertz, Zeitschr. f. Instrumentenk. 3. p. 17. 1883; Schriften 1. p. 227.1895 . 
gummi, $a_{1}$ bis $a_{4}$, angesetzt; in zwei derselben, $a_{1}$ und $a_{2}$, sind längere Glasröhren $r_{1}$ und $r_{2}$, in die beiden anderen, $a_{3}$ und $a_{4}$, kürzere Röhren $r_{3}$ und $r_{4}$ eingekittet. Auf die freien Enden einer jeder dieser Röhren sind Messingkappen (vgl. die auf Röhre $r_{3}$ sitzende, welche im Querschnitt gezeichnet ist) aufgekittet, welche axial cylindrisch durchbohrte, aussen mit Gewinde versehene und längsgeschlitzte Fortsätze tragen; auf dieselben sind flache Muttern aufgeschraubt, mittels deren bei $r_{1}$ und $r_{2}$ die mit Klemmschrauben versehenen Messingstäbchen $s_{1}$ und $s_{2}$, bei $r_{3}$ und $r_{4}$ die Kugeln tragenden Stäbchen $t_{1}$ und $t_{2}$ in jeder beliebigen Stellung innerhalb der Fortsätze festgeklemmt werden können. An die Stäbe $t_{1}$ und $t_{2}$ sind die kurzen Stahldrähte $d_{1}$ und $d_{2}$ gelöthet, welche an dem einen Spiegel $S$ tragenden cylindrischen Messingstabe $T$ genau axial befestigt sind. An dem Stabe $T$ angelöthet und einmal um ihn herumgeschlungen sind ferner zwei je $19 \mathrm{~cm}$ lange Constantandrähte $c_{1}$ und $c_{2}$ von $0,03 \mathrm{~mm}$ Dicke, welche mit ihren anderen Enden an $s_{1}$ und $s_{2}$ angelöthet sind.

Der ganze Apparat ist mittels der Messingbügel $b_{1}$ bis $b_{4}$ auf einem starken Brette befestigt, welches an der Wand festgebolzt ist.

Durch Drehen der Stäbe $t_{1}$ und $t_{2}$ kann man den Drähten $d_{1}$ und $d_{2}$ eine gewisse Torsion und dadurch den Drähten $c_{1}$ und $c_{2}$ eine bestimmte Spannung ertheilen; durch Einreguliren an $s_{1}$ und $s_{2}$ kann man ferner dem Spiegel $S$ eine bestimmte Stellung geben. Bei diesen Manipulationen wird man sehr wesentlich dadurch unterstützt, dass man die Messingstäbchen durch die Flachmuttern genau in der Stellung fixiren kann, in die man sie beim Einreguliren gebracht hat. Schickt man durch $s_{1}$ und $s_{2}$ den hochfrequenten Strom, so wird der Constantandraht warm und dehnt sich aus; die Torsionskraft der Stahldrähte $d_{1} d_{2}$ dreht den Messingstab und mit ihm den Spiegel um einen entsprechenden Betrag herum. Bei einem Scalenabstande von 2,44 $\mathrm{m}$ entsprach bei dem hier beschriebenen Instrumente einem Scalentheile (mm) ein Strom von 1,73 Milliampère.

Die Einstellung ist vollkommen aperiodisch. Die Pulsationen des Wechselstromes erfolgen so rasch. dass der Spiegel vollkommen ruhig steht und ganz scharfe Bilder giebt 
(w̧enn Erscbütterungen fern gebalten werden). Das Instrument konnte direct vor der Entladungsröhre in den secundären Kreis des Transformators gelegt werden, ohne dass irgend welche Veränderung in dem Aussehen der Entladung merklich wurde; der Widerstand des ganzen Drahtes betrug $270 \Omega$, seine Inductanz nur ca. $730 \mathrm{~cm}$.

Da Glas und Constantan nicht den gleichen linearen thermischen Ausdehnungscoefficienten haben, so war die Nullage während einer längeren Beobachtungsreihe nicht ganz constant; es wurden daher immer die Differenzen der Scalenwerthe unmittelbar vor und während der Entladung bei jedem einzelnen Versuche abgelesen; die Wurzeln aus diesen Differenzen mit 1,73 multiplicirt gaben die effective Stromstärke in Milliampères, welche während der Dauer der Entladung sehr nahe constant blieb.

\section{Das Voltmeter.}

Zur genauen Charakterisirung der das verdünnte Gas zum Leuchten anregenden electrischen Schwingung war die Kenntniss der an den Electroden herrschenden effectiven Spannungsdifferenz in allen einzelnen Fällen unerlässlich. Daher musste dem Entladungsrohre ein geeignetes Voltmeter dauernd parallel geschaltet werden; denn es hatte sich gezeigt, dass es durchaus unzulässig ist, die Maximalamplitude etwa aus der Länge einer parallel geschalteten Funkenstrecke zu erschliessen, wie dies gelegentlich auch beim Arbeiten mit electrischen Schwingungen geschehen ist.

Besondere Versuche hatten aber weiter ergeben, dass sowohl irgendwelche Nebenschlüsse zum Rohre, wie auch parallel geschaltete grosse Inductanzen oder Capacitäten das Leuchtphänomen erheblich stören und die Einzelheiten der Erscheinung wesentlich verändern. Die Art dieser Abänderungen ist sehr bemerkenswerth und soll noch einem näheren Studium unterworfen werden, da sie vielleicht Aufschluss über die eigenthümlichen, von Tesla den leuchtenden Gasen zugeschriebenen Inductanzeigenschaften, oder über die obenerwähnten Condensatorwirkungen einer Entladungsröhre, geben dürfte. Hier genügte es, die Bedingungen festzustellen, welche ein Spannungsmesser erfüllen muss, welcher den Einfluss der Schwingungen 
auf das Gas in keiner Weise stört und doch eine genügende Genauigkeit darbietet. Auszuschliessen waren aus den genannten Gründen von vornherein die gewöhnlichen Hitzdrahtvoltmeter, welche immer etwas, wenn auch nur wenig Strom consumiren, sowie alle Anordnungen mit Spulen. Als sehr zweckmässig hat sich eine Anordnung erwiesen, wie sie ähnlich schon von Hrn. V. Bjerknes ${ }^{1}$ ) zum Studium electrischer Schwingungen benutzt wurde: Zwischen zwei kleinen, mit den Punkten, zwischen denen die Spannung gemessen werden soll, verbundenen Platten hängt à einem Quarzfaden unter $\mathbf{4 5}^{\circ}$ gegen die Platten geneigt ein mit Ablesespiegel versehenes Aluminiumblatt. Der Wurzelwerth ans der Winkeldrehung desselben giebt ein Maass für den effectiven Werth harmonisch mit der Zeit variirender Spannungen. In der Form, wie es Hr. Bjerknes benutzte, war das Instrument für den vorliegenden Zweck freilich noch nicht brauchbar. Mein Assistent, Hr. M. W. Hoffmann, und ich haben eine Voltmeterconstruction auf diesem Principe ausgearbeitet, welche allen Anforderungen genügt und sich bei einem Gebrauche während eines Jahres sehr gut bewährt hat. ${ }^{2}$ ) Der hier vor allem in Betracht kommende Vortheil dieses Instrumentes liegt darin, dass es mit vollkommen ungeschlossenem Strome arbeitet und eine Capacität hat, welche selbst gegen diejenige kleiner Entladungsröhren vollkommen verschwindet. Wir haben uns auch davon überzeugt, dass das Instrument noch functionirt, wenn man es an Stelle des etwas subtilen Quarzfadens mit einem dünnen Metalldrahte, der das Aluminiumblatt trägt, versieht.

\section{Versuche.}

1. Aussehen der Gasentladung bei Erregung durch hochfrequenten Wechselstrom. - Ehe auf die quantitativen Beziehungen eingegangen werden soll, mögen einige Bemerkungen allgemeinerer Art vorausgeschickt werden. Um die Bedingungen möglichst einfach und übersichtlich zu gestalten, wurden vorwiegend Entladungsröhren der einfachsten Form verwendet;

1) V. Bjerknes, Wied. Ann. 48. p. 594. 1893.

2) Vgl. H. Ebert u. M. W. Hoffmann, Zeitschr. f. Instrumentenk. 18. p. 1. 1898, woselbst die nähere Beschreibung sowie Angaben über Empfindlichkeit, Messbereich etc. gegeben sind. 
enge Capillaren waren zu vermeiden, weil in ihnen die Erhitzung sehr erheblich wurde, sodass die Gefahr bestand, dass Dämpfe und Verunreinigungen von den Glaswänden losgelöst wurden.

Weil verhältnissmässig grosse Mengen von Energie zugeführt wurden, so mussten auch die Electroden grosse Flächen haben.

Am besten bewährten sich weite, cylindrische, an den Enden völlig symmetrisch gestaltete Röhren mit kreisscheibenförmigen Aluminiumelectroden; im Folgenden wurde verwendet:

Röhre $A, 40 \mathrm{~cm}$ lang, $2,5 \mathrm{~cm}$ weit, mit Electroden von $2,0 \mathrm{~cm}$ Durchmesser in 33,9 cm gegenseitigem Abstande;

Röhre $B$, ebensoweit, aber nur $22 \mathrm{~cm}$ lang; die völlig gleich beschaffenen Electroden waren nur $16,8 \mathrm{~cm}$ voneinander entfernt, also war der Electrodenabstand hier sehr nahe nur halb so gross wie bei $A$;

Röhre $C$; um auch complicirtere Röhrenformen unter der Wirkung des Wechselstromes untersuchen zu können, waren bei dieser Röhre $C$ an $8 \mathrm{~cm}$ weite, kugelförmig gestaltete Electrodenräume mit je einer Aluminiụmscheibe von $2,5 \mathrm{~cm}$ Durchmesser verschieden lange Stücke verschieden weiter Röhren in symmetrischer Anordnung angesetzt und zwar zwei Stücke $30 \mathrm{~cm}$ lang, $1,0 \mathrm{~cm}$ weit, zwei weitere $27 \mathrm{~cm}$ lang, $1,8 \mathrm{~cm}$ weit, zwei Stücke $44 \mathrm{~cm}$ lang, $0,5 \mathrm{~cm}$ weit, zwei Stücke $25 \mathrm{~cm}$ lang und $2,9 \mathrm{~cm}$ weit und ein dazwischen liegendes $52 \mathrm{~cm}$ langes, 1,0 cm weites Verbindungsstück, sodass der Electrodenabstand $304 \mathrm{~cm}$ betrug. Damit die über $3 \mathrm{~m}$ lange Röhre nicht zu unhandlich wurde, war sie U-förmig gebogen, sodass die Electrodenräume dicht nebeneinander zu liegen kamen.

Evacuirt wurde theils mit einer automatischen Pumpe nach Raps von Stuhl in Berlin, theils mit einer TöplerHagen'schen Pumpe von Geissler's Nachfolger in Bonn; die Drucke wurden mit einem einfachen Mac Leod-Manometer, später mit einem solchen Kahlbaum'scher Construction von $\mathrm{Kramer}$ in Freiburg gemessen.

Das Entladungsbild war in allen Fällen ein vollkommen symmetrisches; an beiden Electroden erschienen die drei Kathodenschichten (die der Electrode unmittelbar anliegende, bei Luft orangefarbene Schicht, der [Hittorf'sche] Dunkel- 
raum und die bei Luft bläuliche Glimmlichtschicht). Das Anodenlicht war von der Electrode scheinbar ganz verschwunden. Dasselbe lagerte, bei Luft röthlich gefärbt, zwischen den Glimmlichtern. Dabei war das von allen einzelnen Theilen ausgesandte Licht ein ausserordentlich intensives; bei dem Rohre $C$ ist die gesammte ausgegebene Lichtmenge schon völlig mit dem einer 16 kerzigen Glühlampe vergleichbar. Dabei ist das Licht völlig ruhig. Das lästige Flackern, welches das Studium der Inductoriumentladungen so sehr ermüdend und unsicher macht, fällt hier ganz fort. Aus beiden Gründen eignet sich die vorliegende Art, Gase zum Leuchten zu bringen, vorzüglich auch zu spectralanalytischen Studien oder zar Herstellung homogener Lichtarten durch Ausblenden bestimmter Linien, z. B. der grünen $\mathrm{Hg}_{a}$, welche bei niederen Drucken in Gegenwart einiger Tropfen Quecksilber sehr intensiv wird.

Schichten bilden sich bei den überall gleichweiten cylindrischen Röhren erst unterhalb eines gewissen Druckes aus; in Röhre $C$, welche ungleich weite Theile besitzt, sind sie bei allen Drucken vorhanden. Auch die Schichten stehen vollkommen rubig, sodass man sehr scharfe Photogramme erbält, die man mit grosser Sicherheit ausmessen kann. Die Schichtabstände gehorchen hier nicht dem einfachen Gesetze, welches Hr. Goldstein für den Fall verschieden weiter Theile desselben Entladungsrohres bei verschiedenen Gasdrucken aufgestellt hat. ${ }^{1}$ )

In dem Entladungsbilde lagern sich zwei Einzelentladungen von entgegengesetzter Richtung übereinander. Die Analyse im Drehspiegel giebt folgende weitere Aufschlüsse:

a) Jede Entladung ist ein völlig einheitliches Gebilde; Partialentladungen sind bei den genannten Röhren nicht zu erkennen.

b) Die einzelnen Entladungsbilder sind durch völlig schwarze, wenn auch äusserst schmale Zwischenräume voneinander getrennt, die Entladungen sind also discontinuirlich und die luminescirenden Gase zwischen den einzelnen Entladungen ganz dunkel. Nur bei tiefen Drucken, bei denen auch die innerste, der Electrode unmittelbar anliegende sogenannte erste

1) E. Goldstein, Sitzungsber. d. k. Akad. d. Wissensch. zu Berlin. p. 876. 1881 . 
Kathodenschicht deutlich entwickelt ist, scheinen die Electroden continuirlich mit Licht bedeckt zu sein. Es lässt sich dann erkennen, was schon bei höheren Drucken angedeutet ist, dass das auf der Electrode unmittelbar aufsitzende Anodenlicht in das Licht der ersten Kathodenschicht übergeht. Dies stimmt mit den Angaben anderer Forscher überein, welche darauf hindeuten, dass diese erste Glimmlichtschicht einen Rest von Anodenlicht darstellt, oder diesem doch nahe verwandt ist. Nachdem Hr. Graham gezeigt hat, dass innerhalb des Hittorf'schen dunklen Raumes unmittelbar vor der Electrodenflïche noch einmal ein deutlich ausgesprochener Vorzeichenwechsel im Potentialgradienten eintritt, wird diese eigenthümliche Erscheinung verständlicher. Auch das Spectrum weist bei allen Gasen, die ich daraufhin untersucht habe, auf eine nahe Verwandtschaft mit dem Anodenlichte hin.

Bei der Erregung durch hinreichend rasche electrische Schwingungen bleibt daher die Anodenerscheinung auf der Electrode selbst deshalb unsichtbar, weil sie mit der ersten Kathodenschicht in ihrer Ausbreitung zusammenfällt, also bei der Debereinanderlagerung vollständig durch diese verdeckt wird. Dass aber dieses Licht auch nach erfolgtem Zeichenwechsel, wenn also die betreffende Electrode wieder positiv geworden ist, wesentlich auf diese eng begrenzte Schicht zusammengedrückt bleibt und sich nicht in den Raum hinein verbreitet, der vorher von dem dunklen Kathodenraume eingenommen war, deutet auf eine Nachwirkung der Kathodenphänomene über die Dauer ihrer sichtbaren Erscheinung hinaus, für die ich demnächst noch weitere Anhaltspunkte zu erbringen hoffe.

2. Energieconsum, Leistung und Wirkungsgrad des Gleichstromwechselstrom-Umformers. - Von den vielen Fragen quantitativer Natur, $\mathrm{zu}$ deren Inangriffnahme die hier getroffene Anordnung geeignet erscheint, verdient in erster Linie diejenige nach der bei der Entladung consumirten electrischen Energie Beachtung. Wenn auch für gleichgerichtete Entladungen gelegentliche Angaben hierüber vorkommen, für electrische Schwingungen liegen meines Wissens nur einige wenige Bestimmungen von Hrn. E. Wiedemann und mir selbst ${ }^{1}$ ),

1) H. Ebert u. E. Wiedemann, Wied. Ann. 49. p. 23.1893. 
sowie von Hrn. Spiess vor. $\left.{ }^{1}\right)$ Die aus den erstgenannten sich ergebenden Werthe können ihrer Ableitung nach nur als vorläufige Schätzungen der Grössenordnung gelten; letztgenannte Untersuchungen beziehen sich auf den Fall von Entladungen eines Teslasystems, also auf Entladungsbedingungen, welche von den hier zu Grunde gelegten erheblich abweichen. Es genügt aber nicht, den in das Entladungsrohr selbst hineingegebenen und hier in andere Energieformen umgesetzten Wattconsum $\mathrm{zu}$ messen; um das Zusammenwirken aller einzelnen Theile der Anordnung übersehen zu können, müssen wir der Energietransformation von Anfang an folgen und beginnen daher mit dem dem System zugeführten Gleichstromeffecte.

Die folgende Tabelle enthält die Ergebnisse zweier Messungsreihen über den Wirkungsgrad $n$ des GleichstromwechselstromUmformers und zwar auch ausserbalb der Grenzen, in denen er bei dem Studium der Gasentladungen vorwiegend benutzt wurde. Dabei war die Maschine im Wechselstromkreise durch verschiedene inductionsfreie Widerstände belastet ${ }^{2}$ ).

Aus diesen Vorversuchen war zu ersehen, in welcher Weise der Wirkungsgrad zunahm, je kleiner die Belastung gewählt wurde, und dass bei einer gegebenen Belastung der Wirkangsgrad sich steigern liess, wenn höhere Voltzahlen zum Betriebe verwendet wurden. In Kiel wurde die Maschine mit Accumulatorenstrom gespeist; meist wurde mit 55 Volt gearbeitet; in München liess sich dio Maschine direct an die Lichtleitung der Centrale der k. Technischen Hochschule anschliessen, die dauernd 64 Volt hielt. Der gewöhnliche Stromconsum betrug dann 1,5 bis 2,0 Ampère. Bei den üblichen Preisen für electrische Energie (Kilowattstunde 60 Pfennige) sind also die Consumkosten rund $7 \mathrm{Pfg}$. pro Stunde, also sehr gering. Ein Inductorium z. B. arbeitet viel unökonomischer, schon weil es nicht direct an die Centrale angeschaltet werden kann, und man daher vorher Spannung vernichten muss. F'erner fällt der Unterbrecher und die durch ihn bedingte Energieverwüstung fort.

1) P. Spiess, Inaug.-Diss. p. 46. Kiel, 1896.

2) Die Messungen wurden in dem Versuchslaboratorium des Hrn. Hummel in München angestellt. 
Tabelle 1 .

\begin{tabular}{|c|c|c|c|c|c|c|}
\hline \multicolumn{2}{|c|}{ Gleichstrom } & \multicolumn{5}{|c|}{ Wechselstrom } \\
\hline Ampère & Watt & Ohm & Volt & Watt & Ampère & $n$ \\
\hline
\end{tabular}

1. Bei 50 Volt Gleichstrom:

\begin{tabular}{l|l||r|l|l|l|l}
1,05 & 52,5 & 2385 & 33 & 0,46 & 0,014 & 0,009 \\
1,07 & 53,5 & 1193 & 33 & 0,9 & 0,028 & 0,017 \\
1,07 & 53,5 & 545 & 33 & 2 & 0,06 & 0,038 \\
1,08 & 54 & 350 & 33 & 3,1 & 0,095 & 0,058 \\
1,08 & 54 & 207 & 32 & 4,9 & 0,155 & 0,09 \\
1,1 & 55 & 145 & 32 & 7 & 0,22 & 0,13 \\
1,13 & 56,5 & 113 & 32 & 9 & 0,28 & 0,16 \\
1,2 & 60 & 70 & 31 & 13,8 & 0,45 & 0,23
\end{tabular}

2. Bei 60 Volt Gloichstrom:

\begin{tabular}{l|l||r|l|l|l|l}
1,2 & 72 & 2385 & 41 & 0,7 & 0,017 & 0,01 \\
1,22 & 73 & 1193 & 40 & 1,35 & 0,034 & 0,019 \\
1,22 & 73 & 545 & 40 & 3 & 0,074 & 0,04 \\
1,25 & 75 & 350 & 39 & 4,4 & 0,112 & 0,06 \\
1,25 & 75 & 207 & 39 & 7,4 & 0,189 & 0,10 \\
1,26 & 75,5 & 145 & 39 & 10,5 & 0,27 & 0,14 \\
1,27 & 76 & 113 & 39 & 13,2 & 0,35 & 0,17 \\
1,32 & 79,2 & 70 & 39 & 22 & 0,56 & 0,28
\end{tabular}

3. Zusammenwirken des Gleichstromwechselstrom-Umformers mit dem Hochspannungstransformator bei Leerlauf und bei Belastung mit Entladungsröhren. - Um alle Verhältnisse übersehen zu können, wurden in jedem der drei Stromkreise die Spannungen und Stromstärken gemessen, im primären Wechselstromkreise ausserdem der Stromeffect. Hierzu dienten die folgenden Messinstrumente ${ }^{1}$ ):

a) im Gleichstromkreise $\left.\begin{array}{l}\text { Voltmeter } \\ \text { Ampèremeter }\end{array}\right\}$ der Weston-Company,

1) Bei den Messungen selbst hat mich mein College, Hr. C. Heinke, Docent an der technischen Hochschule in München, aufs Freundlichste unterstützt, wofür ich ihm auch öffentlich meinen besten Dank aussprechen möchte. 
b) im Wechselstromkreise

a) im Niederspannungskreise $\left.\begin{array}{l}\text { Voltmeter } \\ \text { Ampèremeter }\end{array}\right\} \begin{gathered}\text { Hitzdrahtinstrumente von Hartmann } \\ \text { undBraun. }\end{gathered}$ Wattmeter, direct zeigendes Weston-Instrument,

$\beta$ ) im Hochspannungskreise

das Plattenvoltmeter p. 774.

das Hitzdrahtampèremeter p. 772.

Maschine ohne Transformator. Bei 64 Volt Spannung an den Gleichstromklemmen und Serienschaltung von Feldmagneten und Anker consumirte die Maschine 1,4 Ampère, also 90 Watt. Diese Arbeit wird hauptsächlich zur Herstellung des Feldes, der Ueberwindung der Reibung und der electromotorischen Gegenkraft im rotirenden Anker verwendet. Wenn auch die Tourenzahl der Maschine nicht völlig von der Belastung unabhängig war, so können wir doch voraussetzen, dass diese Arbeit in allen folgenden Fällen etwa dieselbe ist, und sie daher von der gesammten zum Betriebe nöthigen Wattzahl abziehen um die Wechselstromleistung zu erhalten.

Maschine mit offenem Transformator. Verwendet wurde Transformator b, p. 771. Es ergaben sich

a) im Gleichstromkreise

64 Volt 1,5 Ampère, also 96 Watt;

b) im Wechselstromniederspannungskreise 36,5 Volt, 0,265 Ampère und 1,25 direct angezeigte Watt.

Zur Erregung des Wechselstromes in dem primären Kreise des Transformers müssen also der Maschine ca. 6 Watt Gleichstrom mehr als bei Nichtbelastung zugeführt werden. Die Leerlaufarbeit 1,25 wird erstens in Joule'sche Wärme verwandelt; der hierauf entfallende Betrag ist, da der Widerstand der Primärwickelung nach p. 771 gleich $1,142 \Omega$ ist, 0,080 Watt. Der Rest von 1,17 Watt wird auf Hysteresisarbeit verwendet. Diesen Betrag müssen wir im Folgenden von der thatsächlich am Wattmeter beobachteten und um die entsprechende Joule'sche Wärme verminderten Wechselstromarbeit abziehen, um den zur Transformation wirklich zur Verfügung stehenden Vorrath an Energie zu erhalten. Derselbe ist aber bei den verschiedenen Belastungen nicht genau der gleiche, schon weil die Periodenzahl etwas schwankt. Mit hinreichender Genauigkeit können 
wir ihn aber bei den folgenden Versuchen zu rund 1 Watt annehmen.

Das Product der beobachteten Spannung und Stromstärke ist gleich 9,67 , also erheblich grösser als der thatsächlich beobachtete Effectwerth 1,25. Dies ist ein Zeichen, dass eine sehr erhebliche Phasendifferenz zwischen Strom und Spannung auftritt, die, wenn sie genau $90^{\circ}$ betrüge, den Wechselstrom zu einem wattlosen Strom machen würde. Aus der Beziehung

$$
A_{\text {beobachtet }}=v_{\text {eff. }} \times J_{\text {eff. }} \cos \varphi
$$

können wir auf Grund der Beobachtungsdaten die Phasenverschiebung berechnen; sie ergiebt sich zu

$$
\varphi=82^{\circ} 34^{\prime} 4 \text {. }
$$

Wattverbrauch in der Hochspannungsleitung. Als die mit Kautschuk überzogenen, ausserdem noch durch Glasröhren geschützten, zum Theil an der Wand entlang geführten $\mathrm{Zu}$ leitungen zum Vacuumrohr angeschlossen wurden, sanken die Werthe für $v$ und $J$ im Niederspannungskreis auf 36,0 bez. 0,23 . Der Wattverbrauch stieg um 1,59; wenn ausserdem das Plattenvoltmeter angeschlossen wurde um 1,69 Watt, Stromstärke und Spannungen blieben ungeändert 0,23 bez. 36,0 . Es sind also bei allen folgenden Messungen ausser den obengenannten $\mathrm{Ab}$ zügen immer noch diese 1,7 Watt in Abrechnung zu bringen.

Maschine mit Transformator, der auf. Entladungsröhren arbeitet. Ich führe hier zunächst die Versuche mit dem p. 771 erwähnten Messtransformator an, weil bei ihm das Uebersetzungsverhältniss genau bekannt war $(1: 20)$. Bei den beiden cylindrischen gleichweiten mit verdünnter trockener Luft gefüllten Entladungsröhren $A$ und $B$ ergaben sich bei demselben Gasdruck (Dicke des Kathodendunkelraumes etwa 2,5 mm) die folgenden Zahlen:

\begin{tabular}{|c|c|c|c|c|c|c|c|c|c|c|c|}
\hline \multirow{3}{*}{ 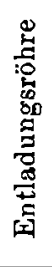 } & \multirow{2}{*}{\multicolumn{3}{|c|}{ Gleichstrom }} & \multicolumn{8}{|c|}{ Wechselstrom } \\
\hline & & & & \multicolumn{5}{|c|}{ Niederspannungskreis } & \multicolumn{3}{|c|}{ Hochspannungskreis } \\
\hline & $\stackrel{\circ}{\circ}$ & $\underset{\square}{\stackrel{D}{D}}$ & 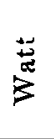 & $\stackrel{2}{\circ}$ & 惫 & 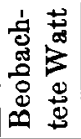 & $\begin{array}{l}\frac{4}{0} \\
\stackrel{5}{0} \\
x\end{array}$ & 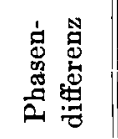 & $\frac{7}{\circ}$ & 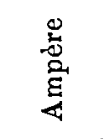 & 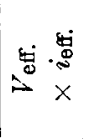 \\
\hline$A$ & 64 & 1,62 & 104 & 35,0 & 0,61 & 12,0 & 21,3 & $55^{0} 42,5^{\prime}$ & 740 & 0,0068 & 5,0 \\
\hline$B$ & 64 & 1,68 & 108 & $3 \overline{\mathbf{s}}, 0$ & 0,65 & 19,3 & 22,7 & $31^{\circ} 45^{\prime}$ & 710 & 0,0119 & 8,5 \\
\hline
\end{tabular}

Tabelle 2. 
Bemerkenswerth ist hierbei vor allem, dass die Secundärspanmungen in beideu Fällen etwas höher sind, als dem Transformationsverhältnisse entsprechen würde. Dies ist ein Anzeichen dafür, dass auch in dem secundären, das Entladungsrobr enthaltenden Kreise eine Phasendifferenz $\varphi^{\prime}$ Platz greift. Ihre Grösse wollen wir aus späteren Beobachtungen ableiten; wichtig ist zunächst, den Sinn der Verschiebung zwischen Strom und Spannung festzustellen. Zeichnet man das entsprechende Vectorendiagramm, welches hier, um Platz zu sparen, weggelassen ist, so erkennt man, dass der Strom der Spannung vorauseilt. Dies deutet darauf hin, dassis ein Entladungsrohr den Schwingungen gegenüber wie ein Condensator wirkt. Die Condensatorwirkung ist je nach der Entwickelung der Dunkelräume eine verschiedene (vgl. w. u. p. 788).

4. Energieverbrauch in verschiedenen Entladungsröhren bei verschiedenen Drucken. - Die Belästung, welche eine in den Hochspannungskreis eingeschaltete Entladungsröhre herbeiführt, ist je mach ihrer Gestalt, Grösse und dem Gasdruck, sowie der Natur des leuchtenden Gases eine sehr verschiedene. Einen Ueberblick hierïber giebt die Tab. 3.

Zur Charakterisirung des Gasdruckes wurde einfach die Dicke $d$ des Kathodendunkelraumes verwendet.

Da 96 Watt die Leerlaufarbeit der an den Transformator angeschlossenen Maschine war, so erkennt man, dass in den vier hier aufgeführten Fällen 18, 16, 16 bez. 20 Watt mehr in die Maschine eingeführt werden mussten, um das Entladungsrohr zu speisen. Dass im letzten Falle die im primären Wechselstromkreise erscheinende Arbeit von 27,0 Watt die Leerlaufarbeit von 1,25 (rgl. oben p. 781) um ca. 26 Watt übertrifft, also hier scheinbar $6 \mathrm{Watt}$ mehr auftreten, als dem Gleichstromkreise mehr zugeführt wurden, enthält keinen Widerspruch gegen das Energieprincip; in diesem Falle war die Maschine etwas günstiger belastet und ihr Wirkungsgrad war erhöht, sodass die obige Zahl für die Leerlaufarbeit hier keine Anwendung mehr findet. Die wirklich zur Transformation gelangende Wattzahl (10. Columne) wird erhalten, wenn man von den direct gemessenen primären Watt (7. Columne) die auf Joule'sche Wärme in der Primärwickelung und das eine auf Hysteresisarbeit verwendete Watt (p. 784) abzieht. 


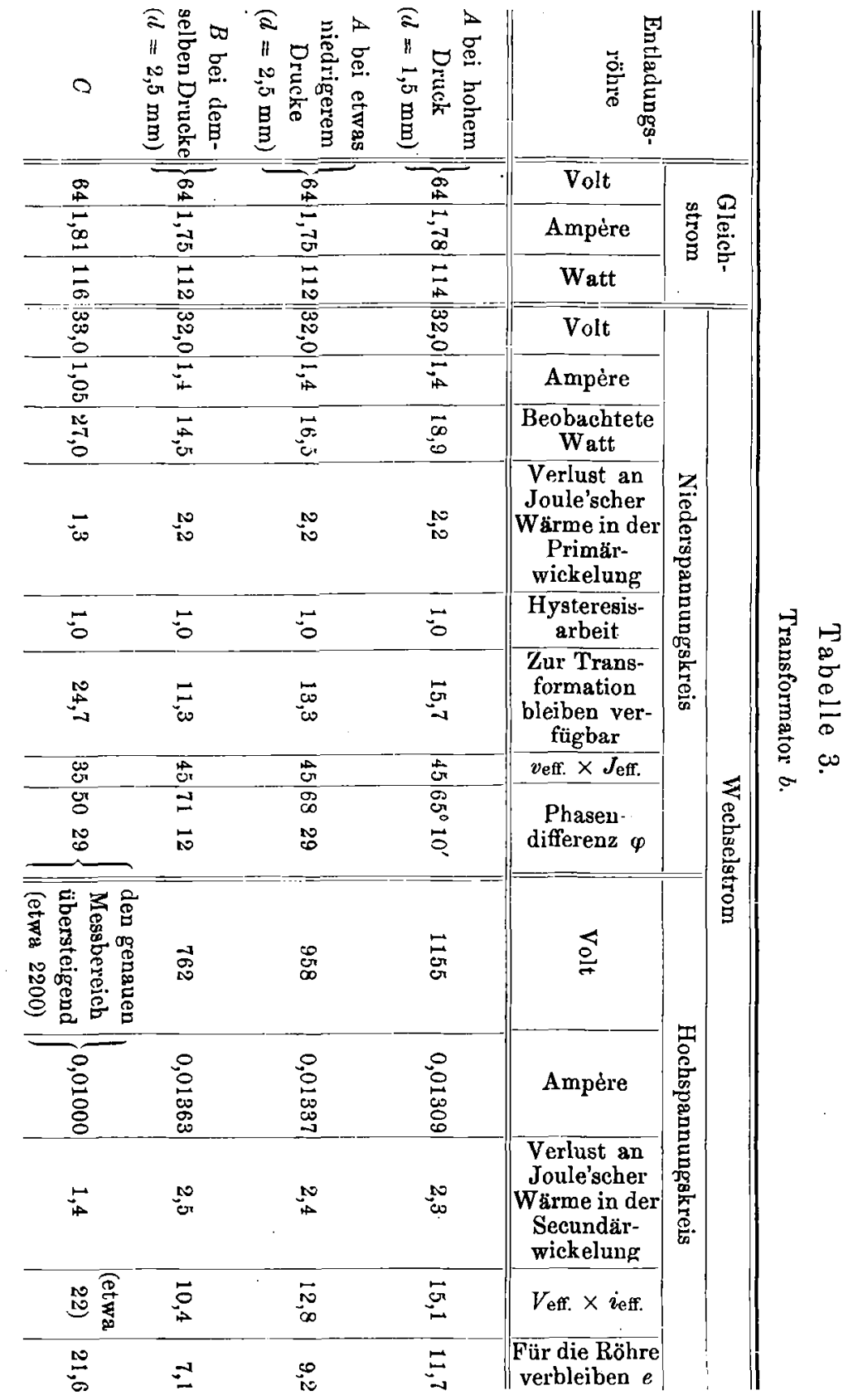


Von dem transformirten Effect ist erstens die in der Secundärwickelung auftretende Joule'sche Wärme abzuziehen und zweitens die 1,7 Watt, welche nach p. 782 in der Leitung verloren gehen. Auf diese Weise werden die Zahlen der letzten Columne erhalten, welche den electrischen Effect $e$ angeben, welcher wirklich auf das Entladungsrohr wirkt. Er ist bei dem langen Rohre $C$ sehr gross; hier wird ein sehr grosser Theil der überhaupt transformirten Energie zum Erregen des Rohres selbst verwendet; bei kürzeren Entladungsröhren ist dieses Verhältniss ungünstiger; die Stromstärke ist hier erheblicher, ein grosser Theil der Energie erwärmt die secundäre Wickelung. Man sieht aber auch, dass der Energieconsum eines Entladungsrohres wesentlich vom Gasdrucke abhängt. Um über den Gang dieser Erscheinung genaueren Aufschluss zu erhalten, habe ich ein grosses Beobachtungsmaterial gesammelt, das ich demnächst gesondert zu publiciren gedenke. Hier mögen die folgenden Andeutungen genügen.

Sowohl bei bohen Drucken, bei denen die Gassäule eben durchbrochen wird, und die Entladung einsetzt, wie bei sehr niederen ist die Spannung an den Electroden sehr hoch, die Stromstärke gering; bei einem gewissen mittleren Drucke erreicht erstere ein Minimum, letztere bei demselben Gasdrucke ein Maximum. ${ }^{1}$ ) Hand in Hand damit geht bei abnehmendem Drucke (nach einer kleinen Steigerung bei hohem Drucke) zunächst ein Abnehmen des Productes der Volt und Ampères bis zu jenem Umkehrpunkte hin, dann ein Zunehmen ${ }^{2}$ ) bis zu einem Maximum; bei sehr niedrigen Drucken, bei denen schliesslich gar keine Entladungen durch das Rohr mehr hindurchgehen, nimmt dann die verbrauchte Energie wieder bis zu Null ab.

1) Ein solches gleichzeitiges Umkehren von Spannung und Stromstärke haben auch die Herren $\mathrm{H}$. Paalzow und F. Neesen bei ihren Untersuchungen der Batterieentladungen durch verdünnte Gase beobachtet (Wied. Ann. 56. p. 289. 1895).

2) Dieses Abnehmen der electrischen Energie bis zu einem Minimum bei einem bestimmten Drucke und dann das Wiederzunehmen des Wattverbrauches geht ganz parallel der Gesammterwärmung des Rohres oder der Erwärmung der Kathode, welch letztere bei dem Ueberwiegen der Kathodenerscheinung in unseren Entladungsröhren das Bestimmende ist; vgl. E. Wiedemann, Wied. Ann. 10. p. 214 u. 226.1880. 
Inwieweit sich die Maschine diesen wechselnden Belastungen anzupassen vermag und die Belastungsschwankungen in dem secundären Transformatorkreise sich auf den Gleichstrom übertragen, zeigt die

Tabelle 4.

Transformator $a$.

\begin{tabular}{|c|c|c|c|c|c|c|c|}
\hline \multicolumn{4}{|c|}{ Gleichstrom } & \multicolumn{3}{|c|}{ Wechselstrom secundär } & \multirow{2}{*}{ Gasdruck } \\
\hline & Volt & Amp. & Watt & Volt & Amp. & $V \times i$ & \\
\hline \multirow{5}{*}{ 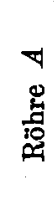 } & 61,0 & 1,7 & 103,7 & 1232 & 0,0000 & & sehr hoch \\
\hline & 61,0 & 1,9 & 115,9 & 779 & 0,0066 & 5,1 & ca. $5,8 \quad \mathrm{~mm}$ \\
\hline & 60,8 & 2,0 & 121,6 & 444 & 0,0116 & 5,2 & 1,34 \\
\hline & 60,9 & 1,9 & 115,7 & 663 & 0,0064 & 4,2 & 0,147 \\
\hline & 61,1 & 1,7 & 103,9 & 1022 & 0,0017 & 1,7 & 0,061 \\
\hline
\end{tabular}

Der oben beschriebene Gang in dem Wattverbrauch der Röhre setzt sich in den Gleichstromkreis hinein fort. Es wird von der Maschine also nichts zwecklos producirt, sie passt sich vielmehr den wechselnden Belastungen vollkommen an. Dabei ist gerade in dem Druckbereiche, in dem die Gasentladungen besonders charakteristische Erscheinungen zeigen, für welchen die Anordnung also hauptsächlich verwendet wird, das Güteverhältniss der Transformation ein ganz besonders günstiges (vgl. auch Tab. 3).

Vor allem geht auch nach Tab. 2, 3 und 4 der Mehrbedarf im Gleichstromkreise herab, wenn etwa durch fortgesetzte Evacuation der Wattverbrauch im Entladungsrohre sich vermindert.

Zur Charakteristik der Leistungen der Gesammtanordnung mögen endlich noch die folgenden Angaben dienen: Wurde die secundäre Leitung des Transformators, welche, wenn sie offen war, also bei Leerlauf und bestimmter Tourenzahl 1592 Volt Spannung zeigte, durch einen Condensator von 2,80 Mikrof. geschlossen, so ergab sich eine Ladestromstärke von 26,3 Milliamp. bei einer effectiven Klemmenspannung von 66 Volt; dabei wurde die Tourenzahl des Generators durch diese Belastung um mehr als die Hälfte herabgedrückt. Kurzschluss im secundären Kreise ergab 25,7 Milliamp. Stromstärke; die Klemmenspannung war dabei 
so herabgesetzt, dass sie nicht mehr messbar war. Ein Graphitwiderstand ergab die Stromstärke Null; die Spannung war dieselbe wie bei offenem Kreise.

5. Berechnung der Phasendifferenz zwischen Strom und Spannung in einem durch electrische Schwingungen erregten Entladungsrohre. - Das Product von Spannung und Stromstärke giebt bei einem von Wechselströmen durchflossenen System nur dann die in demselben vorhandene Energie, wenn die Phasendifferenz zwischen den beiden genannten Grössen Null ist. Bereits oben (p. 783) wurde aber darauf hingewiesen, dass dies bei einer Entladungsröhre offenbar nicht der Fall ist; ein leuchtendes Gas leitet zwar, es leitet, wie es scheint, auch wie ein Leiter von nicht grösserer oder kleinerer Selbstinduction, als der geometrischen Gestalt der Leitungsbahn und der magnetischen Permeabilität des umgebenden Mediums entspricht, es wirkt aber auf einen Wechselstrom wie ein Leiter mit nicht unerheblicher Capacität. Durch dieselbe tritt eine Phasenverschiebung $\varphi^{\prime}$ auch in dem secundären Kreise und zwar derart ein, dass die Stromwelle in der Phase vorgezogen wird und die Spannungswelle zurückbleibt. Die Zahlen der Tab. 3 gestatten $\varphi^{\prime}$ zu berechnen, da wir das Product der Effectivwerthe einerseits, die dem Rohre selbst zugeführte Effectivgrösse andererseits kennen. Die untenstehende Tabelle 5 giebt die Resultate, die Folgendes erkennen lassen:

- Die Phasendifferenz wächst (innerhalb des untersuchten Druckintervalles) mit abnehmendem Drucke; sie ist bei der kurzen cylindrischen Röhre $B$ grösser als bei der gleichweiten doppelt so langen Röhre $A$; bei der sehr langen Röhre $C$ ist sie sehr klein, die Voltampères stellen sehr nahe auch den wirklichen Wattverbrauch dar.

Tabelle 5 .

\begin{tabular}{c|cc}
\hline & \multicolumn{2}{c}{ Phasendifferenz $\varphi^{\prime}$ bei Röhre } \\
\hline $\begin{array}{c}\text { hoher Druck } \\
(d=1,5 \mathrm{~mm})\end{array}$ & $\begin{array}{c}\text { niederer Druck } \\
(d=2,5 \mathrm{~mm})\end{array}$ & $\begin{array}{c}\text { niederer Druck } \\
(d=2,5 \mathrm{~mm})\end{array}$ \\
\hline $39^{\circ} 13^{\prime}$ & $4^{\circ} 3^{\prime}$ & $C$ \\
\hline $46^{\circ} 56^{\prime}$ & sehr klein
\end{tabular}

Dieses Resultat stimmt mit der Thatsache überein, dass die Spannung an den Elektroden zunächst durch einen das 
vorgelegte Hitzdrahtampèremeter passirenden Strom, einen Ladestrom, auf eine gewisse Höhe gebracht werden muss, ehe die Entladung und damit der Spannungsausgleich stattfindet. Das Aufteten der Phasendifferenz $\varphi^{\prime}$ bringt die Condensatornatur eines mit leuchtendem Gase erfüllten Raumes, sowie die Capacitätseigenschaften desselben am deutlichsten zum Ausdruck. - In einer späteren Arbeit soll die Wärmeentwickelung in Entladungsröhren unter der Einwirkung der ungedämpften Schwingungen discutirt und im Anschlusse daran die absolute Bestimmung der Capacitätsgrösse selbst versucht werden.

Die vorliegenden Untersuchungen dürften zeigen, dass der hochfrequente Wechselstrom bei dem Studium der Gasentladungen ein wichtiges Hülfsmittel zu werden vermag. -

Zum Schlusse möchte ich nicht verfehlen, Hrn. Dr. W. Hoffmann für seine Unterstützung bei dieser Arbeit meinen besten Dank zu sagen.

München, Phys. Inst. d. Techn. Hochschule, Juli 1889.

(Eingegangen 12. Juni 1898.) 